\title{
SUSPENSION VIBRATION COMPENSATION USING A MEMS MICROACTUATOR IN HARD DISK DRIVES
}

\author{
Tsung-Lin Chen* Kenn Oldham* Yunfeng Li * \\ Roberto Horowitz ${ }^{*, 1,2}$ \\ * Department of Mechanical Engineering, University of \\ California, Berkeley, CA 94720-1740, USA
}

\begin{abstract}
This paper discusses the design and fabrication of a prototype MEMS microactuator and vibration sensors, and controller design for suspension vibration compensation in disk drives. The microactuator utilizes parallel-plates actuation and comb-finger position sensing. An electrical isolation MEMS fabrication technique is utilized to increase the actuation force. An LQG method is proposed for vibration sensor location and orientation optimization. Strain sensors are fabricated by depositing polysilicon on a metal wafter. A vibration control scheme using adaptive feedforward control is formulated to cancel suspension vibration using the microactuator.
\end{abstract}

Keywords: Disk Drive, Microactuator, Vibration Control, Suspension, Windage

\section{INTRODUCTION}

With the continuing increase in storage density and spindle rotation speed in hard disk drives, airflow excited suspension vibration (windage) becomes a significant challenge for achieving the required track mis-registration (TMR) budget. The idea of actively control suspension vibration modes to expand the servo bandwidth using an instrumented suspension has been proposed by (Huang et al., 1999). Similar idea can be used to suppress the suspension vibration induced TMR using dual-stage actuators.

For suspension vibration compensation using dualstage stage servo systems, the control scheme is dependent on the configuration and location of the second stage mini/micro-actuators. For a

\footnotetext{
1 Research supported by the Computer Mechanics Laboratory (CML) of U.C. Berkeley and the National Storage Industry Consortium (NSIC).

2 Corresponding author E-mail: horowitz@me.berkeley.edu.
}

PZT-actuated-suspension based dual-stage system, the PZT actuators are located behind the suspension vibration modes. A feedback damping control scheme has been shown to be effective in suppressing airflow excited suspension vibration (Li and Horowitz, 2002). For an actuated slider/head dual-stage system with a translational microactuator (MA), suspension vibration can be passively attenuated by the MA if the resonance frequency of the MA is lower than that of the suspension's resonance modes. For an actuated slider/head dual-stage system with a rotational MA, feedforward control can be utilized to cancel the suspension vibration with the MA ( $\mathrm{Li}$ and Horowitz, 2001a).

Several MA designs have been proposed for dualstage actuation in hard disk drives (Fan et al., 1999), (Horsley et al., 1999). The MA design proposed in this paper utilizes parallel-plates actuation scheme for high actuation force output and comb-drive sensing scheme for linear position sensing performance. An electrical-isolation tech- 
nique is adopted in this MA design to increase the driving force and thus lower the driving voltage. For active suspension vibration compensation, vibration sensors need to be attached/fabricated on the surface of the suspension to sense its vibration. High gauge factor vibration strain sensors are fabricated by depositing polysilicon on metal substrate. An adaptive feedforward controller is developed to cancel the suspension vibration induced TMR using the output of the vibration sensor and the MA.

Section 2 of the paper presents the design and fabrication of a prototype MA. Section 3 describes the fabrication of the vibration strain sensor and a method to find the optimal sensor location and orientation. Section 4 discusses the vibration compensation controller design. Conclusions are provided in section 5 .

\section{DESIGN AND FABRICATION OF THE MEMS MICROACTUATOR}

\subsection{Electrostatic Mircroactuator Design}

Electrostatic actuation has been selected for the secondary actuation for ease of fabrication, since the structural material needs only to be conductive, rather than ferromagnetic or piezoelectric. Electrostatic MAs have been widely used in many applications, and are often categorized into two groups, depending on the configuration of their electrodes: comb-drive and parallel-plates. It is a general consensus that the comb-drive actuation scheme is suitable for the applications that require a large actuation stroke and small actuation force. Conversely, the parallel-plates scheme is generally used in applications that require a large force output and small actuation stroke. In addition, from the servo system perspective, the comb-drive actuation is always stable and has a linear force output over the actuation stroke while the parallel-plates actuation is highly nonlinear and may become unstable under large actuation voltages.

Electrical-isolation is another challenge for designing a parallel-plates MA. Generally speaking, when multiple voltage levels are needed in MEMS devices, electrical isolation is achieved by structurally separating the parts that need to be on different voltage levels and anchoring them separately to a non-conductive substrate. This approach has several drawbacks not only because it requires the use of a substrate in the device, but also requires the structures have to be mechanically separated to be electrically isolated. The electrical-isolation problem is far more severe in parallel-plates MAs than in comb-drive MAs since parallel-plates actuation generally requires more voltage levels for operation.
In our dual-stage actuation application, parallelplates MAs were preferred and selected because this application requires the actuator to produce a large force to carry a picoslider (which is 10 times heavier than MA) over a relative small displacement range $(+/-1 \mu \mathrm{m})$. As for the inherent design and performance limitations of parallelplates MAs discussed above, our solutions are: (1) to develop a bulk fabrication process that can efficiently implement electrical-isolation features in parallel-plates MAs, (2) to incorporate capacitive position sensing mechanism in the MA to achieve robust closed loop performance.

Fig. 1 shows a photo of the proposed electrostatic MA design. As indicated in the photo, there are parallel-plates structures along two sides of the MA to generate actuation force and comb-drive structures on the traverse sides for the position sensing. The overall structure has its dimensions of $4.5 \mathrm{~mm} \times 3 \mathrm{~mm} \times 0.08 \mathrm{~mm}$, which can easily fit in a commercially available suspension.

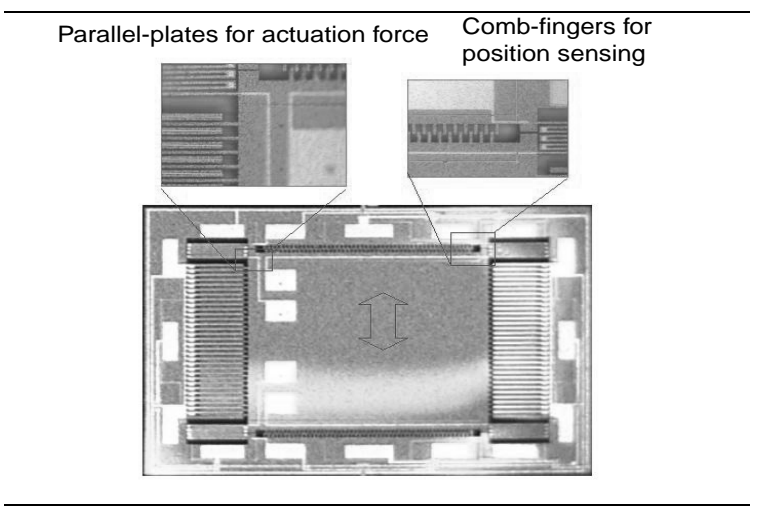

Fig. 1. Electrostatic Micoractuator

Fig. 2 explains how structural "electrical-isolation" has been utilized in this parallel-plates MA design to increase the actuation force output. As shown in the figure, without the proper electrical isolation, drive-electrodes that have different voltage potentials have to be placed in separate groups, and result in a same voltage difference across electrodes and shutter on two sides of each shutter finger (Horsley et al., 1999), (Muller, 2000). Due to the fact that electrostatic force are always attractive, gaps on two sides of the interlaced structure cannot be made equal. Otherwise, opposing forces on two sides of shuttle fingers would be equal and thus the shuttle moving direction would not be controllable. With the use of structural electricalisolation, the gaps of the interlaced structure can be the same size, since the voltage differences on two sides of the shuttle fingers can be different. As shown in Fig. 2, the design which has structural electrical-isolation is more space efficient than one that does not have this feature. Simulation results showed that the force density of a design with structural electrical-isolation can be 3 times higher than a design without it. Furthermore, the 
simulations also indicated that this MA design was able to move a picoslider $+/-1 \mu \mathrm{m}$ at $2 \mathrm{KHz}$ when the MA is biased at 12 Volts and driven at $+/-5$ Volts.

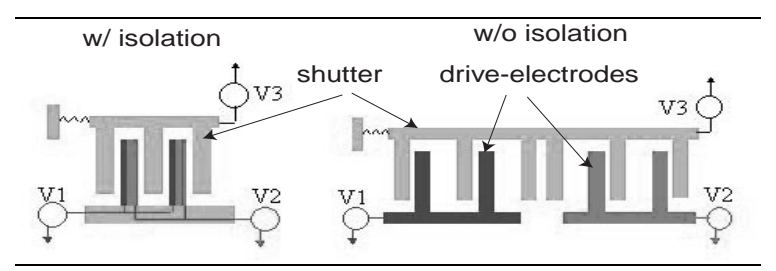

Fig. 2. Parallel-plates MAs and electrical-isolation

Capacitance sensing is another unique features in this MA design. Most of the current electrostatic MA designs acquire capacitance sensing by converting half the driving electrodes to sensing electrodes and thus lose half of their driving capability. By using structural electrical-isolation, we were able to integrate the sensing electrodes on the traverse side of the driving electrodes without compromising force output. Furthermore, this capacitance sensing design will also benefit from the comb-drive scheme, which has a linear capacitance variation output over the actuation stroke.

Some preliminary tests have been performed on the MA. Experimental data shows that the MA is capable of moving a picoslider $+/-1 \mu \mathrm{m}$ with a 20Volts biased voltage and a driving voltage of $+/-8$ Volts. The operation voltage was higher than expected mainly due to the fact that the micromachined gaps between shutter and driving electrodes ended up being $25 \%$ wider than what was designed. Recently, a slightly revised and larger microactuator design, with geometry compatibile with MEMS specific suspensions, has been operated at even lower voltages, with a 12 Volt bias voltage and less than 5 Volt driving voltage, though in this case anticipated driving voltage requirement was just 3 Volts. Again, the gaps were wider than desired and, worse, the depth of these deep trenches was much less than anticipated, resulting in a microactuator that was too fragile for testing over a disk. Better characterization of the deep trench etching process and alteration of the design to devote a larger area to support is underway.

\subsection{Microactuator Fabrication}

The fabrication process is the key to the success to this high performance electrostatic MA. A newly developed bulk fabrication process not only allowed us to integrate structural electrical-isolation features into the electrostatic MA, but also allowed us to fabricate high aspect ratio $(\geq 20) 3 \mathrm{D}$ structures, without using costly SOI wafers. The starting material is a highly conductive, doubledsided polished, 1-0-0 silicon wafer. The process uses an Inductive Coupled Plasma (ICP) system to pattern high aspect ratio trenches. Trenches were subsequently refilled with dielectric material (silicon nitride and undoped polysilicon) to form isolation plugs and finished with a planarized surface topography. The ICP ethcer was utilized again to patterned the mechanical structures of the MA. The structures and trenches are subsequently coated with a thin layer of silicon nitride to protect them from $\mathrm{KOH}$ etcher attack.

The structures are subsequently released by the $\mathrm{KOH}$ etch from the backside of the wafer. The front side is protected by silicon nitride over a planarizing $\mathrm{SiO}_{2}$ layer, which holds the structure in place. The final released is done by a timed $\mathrm{HF}$ etch, which eats away the nitride protection layer and $\mathrm{SiO}_{2}$ from the structure. This release process is an alternative to using SOI wafer. The fabrication process flow is shown in the Fig. 3.

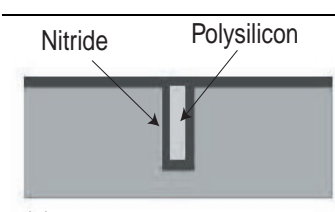

(a) Isolation plugs

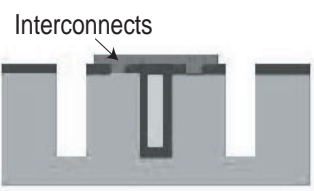

(b) Electrical interconnects (c) Structures paterning

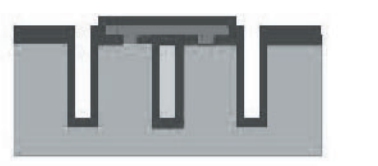

(d) $\mathrm{KOH}$ etch stop: nitride deposition

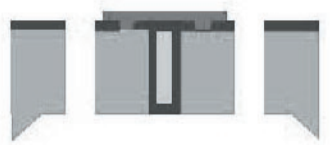

(e) Release: $\mathrm{KOH}$ backside etch
Fig. 3. MA fabrication process flow

\section{DESIGN AND FABRICATION OF THE VIBRATION SENSOR}

\subsection{Fabrication of the Stain sensor}

In order to detect vibration of the disk drive suspension during operation, we have proposed to instrument the suspension with piezoresistive strain sensors, using MEMS-type techniques on a steel, rather than silicon substrate. Specifically, we propose the use of polysilicon as a strain gage material, due to the possibility for higher gage factor than with other strain sensing materials and the availability of low temperature processing equipment. As a test of the procedure, several piezoresistive strain sensors were fabricated on a $60 \mathrm{~mm}$ thick stainless steel substrate. A $0.2 \mathrm{~mm} \mathrm{SiO}_{2}$ film was deposited by plasma-enhanced chemical vapor deposition (PECVD) to insulate the sensors from the substrate, followed by a $1 \mathrm{~mm}$ thick sputtered silicon film to form the piezoresistive layer. The sensors were patterned lithographically and etched by reactive-ion etching (RIE) of the silicon. These sensors were arranged in Whetstone 
bridge configuration at the base of $15 \mathrm{~mm}$ long cantilever beams; the beams were patterned by a second lithography step and cut by wet etching in Ferric Chloride.

The completed sensors were tested under a probe station by measuring voltage change across the bridge as the cantilever beams were deflected. The sensors exhibited a gage factor of 25 to 30, near the upper limit for polysilicon piezoresistivity and an order of magnitude higher than gage factors for metal strain gauge materials. Unfortunately, the resistance of the test sensors was excessive, on the order of several hundred mega-ohms, resulting in extremely large thermal noise levels. This was a result of uncertainty of the doping level of the silicon sputtering source currently available; future sensors will be fabricated from a sputtering source doped to resistivity near $0.05 \mathrm{Ohm}-\mathrm{cm}$. At this dopant level, gage factor is expected to decrease 10 to 20 , but the accompanying reduction in thermal noise will be necessary to resolve the strains present in the suspension during disk drive operation. Additionally, the $\mathrm{SiO}_{2}$ was found to be unreliable as an insulating layer, as it could crack during handling of the flexible steel substrate. A spin-on polymer is expected to prove more resilient and will be used in place of $\mathrm{SiO}_{2}$ in future devices. Using these new materials, the sensors will be fabricated as before on steel substrates, which will be used to produce load beams and assembled into full suspensions at Hutchinson Technology, Inc.

\subsection{Determining the Vibration Sensor Location and Orientation}

The location and orientation of the stain sensors has to be determined so that the output of the strain sensor has optimal correlation with the suspension vibration induced head off-track motion. A numerical search algorithm has been developed to determine the sensor location and orientation which maximizes the minimum singular value of the observability grammian (Huang et al., 1999). However, this method does not consider the relative contribution to the head offtrack motion from different suspension vibration modes, and may not result in good control performance for suppressing suspension vibration induced TMR. In this case, we are using stationary linear quadratic gaussian (LQG) optimization. We model the suspension and microactuator as a linear system with sensor output $z$ and off-track displacement $y$

$$
\begin{aligned}
x(k+1) & =A x(k)+B u(k)+B_{w} w(k) \\
z(k) & =C x(k)+v(k) \\
y(k) & =C_{y} x(k)
\end{aligned}
$$

Disturbance $w(k)$ and noise $v(k)$ are treated as white, zero mean, Gaussian, and uncorrelated. We then minimize the stationary linear quadratic gaussian objective function for a given sensor location

$$
J=E\left[x^{T}(k) C_{y}^{T} C_{y} x(k)+u^{T}(k) R u(k)\right]
$$

with $R$ a weighting function on the control effort. Using LQG results, the value of the objective function can be found in closed form as

$$
J_{\mathrm{inf}}^{o p t}=\operatorname{tr}\left[P_{s}\left(B K_{s} Z_{s} A^{T}+B_{w} W B_{w}^{T}\right)\right]
$$

In the above equation, only the estimation error covariance, $Z_{s}(k)$, is dependent on sensor location, permitting efficient calculation of the objective function value at potential sensor locations. This LQG method of choosing a sensor does a better job of weighing the relative importance of the suspension modes than an optimization based solely on observability, as it locates the sensor to best detect the modes that can be both a significant influence on off-track error and be compensated effectively by the actuator.

\section{VIBRATION COMPENSATION CONTROLLER DESIGN}

For a MEMS MA actuated slider dual-stage servo system, the MA is sandwiched between the gimbal and the slider, and it either translates or rotates the slider relative to the suspension. Depending on the type of the MA (translational or rotational), the dynamics coupling effects of the suspension vibration and the head motion are different. For a dual-stage servo system using a translational MA, suspension vibration is transmitted to the head motion through the spring-damper-mass dynamics of the MA. Thus, if the resonance frequency of the MA is designed to be lower than that of the suspension's resonance modes, the TMR due to suspension vibration can be passively attenuated by the MA, and thus active vibration control may not be necessary.

However, for the dual-stage servo system with a rotational MA, the suspension vibration is directly superposed to the head motion through pivot connection between the MA and the suspension. In this case, the MA can not passively attenuate suspension vibration induced TMR, and we need to compensate suspension vibration through active vibration control implemented using the output of the vibration sensor. Since the vibration sensor attached/fabricated on the surface of the suspension is located in front of the MA, a feedforward control scheme can be used so that motion of the MA cancels the slider off-track motion induced by the suspension vibration. 


\subsection{Adaptive Feedforward Vibration Compensation}

An adaptive feedforward control structure for vibration cancellation is shown in Fig. 4. For simplicity, the dual stage feedback part of the servo architecture is not included in the figure.

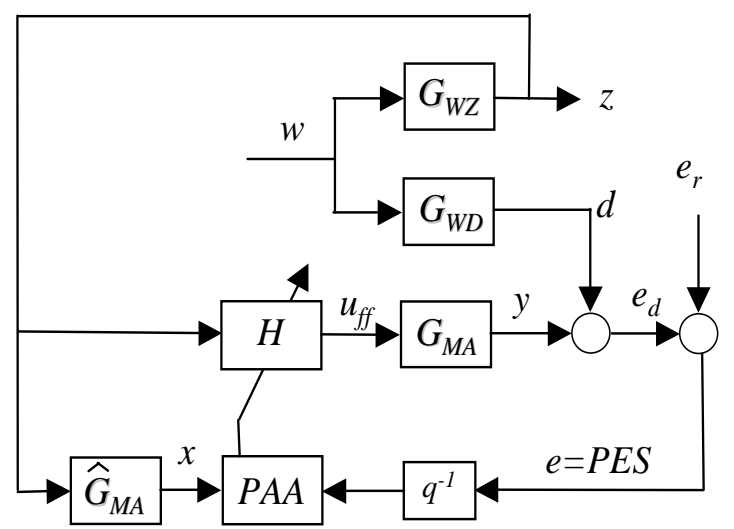

Fig. 4. Adaptive feedforward control for vibration cancellation

Since here we are considering an actuated slider dual-stage servo system with a rotational MEMS MA, suspension vibration induced off-track motion, $d$, is directly added to the MA output $y$.

$w$ in Fig. 4 represents the air flow excitation to the suspension. $G_{w d}$ is the transfer function from $w$ to $d, z$ is the strain sensor output, and $G_{w z}$ is the transfer function from $w$ to $z$. Ideally, we would like $z$ and $d$ to have the biggest possible correlation. However, since $z$ and $d$ represent the effects of vibration at different locations on the suspension, even though the poles of $G_{w z}$ and $G_{w d}$ may be the same, they usually have different zeros, and therefore, $d$ and $z$ will probably have a dynamic relationship. We define the transfer function from $z$ to $d$ to be

$$
G_{z d}=\frac{G_{w d}}{G_{w z}} .
$$

$G_{M A}$ in Fig. 4 is the transfer function of the MA. $H$ is the feedforward filter, which needs to be designed, in order to cancel $d$. Intuitively, an expression for $H$ can be

$$
H=\left(G_{M A}\right)^{-1} G_{z d} .
$$

However, $H$ as given by Eq. (7) may not be causal and the transfer functions of $G_{z d}$ is not precisely known. Furthermore, the strain gauge factor usually is sensitive to the temperature. Thus, the optimal filter $H$ needs to be estimated using an adaptive scheme. In our approach, $H$ is approximated with a finite impulse response (FIR) filter for stability considerations. It can be written as

$$
H\left(q^{-1}\right)=h_{0}+h_{1} q^{-1}+\ldots+h_{M}^{-M} .
$$

where $q^{-1}$ is the one step delay operator and $M$ is the order of the FIR filter. The output of the $\mathrm{MA}$ is

$$
\begin{aligned}
y(k) & =G_{M A}\left(q^{-1}\right) u_{f f} \\
& =G_{M A}\left(q^{-1}\right) H\left(q^{-1}\right) z(k) \\
& =H\left(q^{-1}\right) G_{M A}\left(q^{-1}\right) z(k) .
\end{aligned}
$$

Let

$$
x(k)=G_{M A}\left(q^{-1}\right) z(k),
$$

and define the regressor vector,

$$
\phi(k)=[x(k) x(k-1) \ldots x(k-M)]^{T},
$$

and the FIR tap weight vector,

$$
\theta=\left[h_{0} h_{1} \ldots h_{M}\right]^{T} .
$$

The output equation of the MA can be written as

$$
y(k)=\theta^{T} \phi(k) .
$$

And the vibration compensation error is

$$
e_{d}(k)=y(k)+d(k) .
$$

Parameter adaptation algorithm (PAA) can be used to estimate the coefficients of $H\left(q^{-1}\right), \hat{\theta}$, to minimize the mean square value of $e_{d}, E\left[\left|e_{d}(k)\right|^{2}\right]$. However, $e_{d}$ is not available, and the error signal we have access to is the PES, which can be written as

$$
e(k)=e_{d}(k)+e_{r}(k),
$$

where $e_{r}$ represents the position error from other sources, such as runout, after compensated by the feedback controller. Assuming that $d$ is uncorrelated with $e_{r}$, we have

$$
E\left[|e(k)|^{2}\right]=E\left[\left|e_{d}(k)\right|^{2}\right]+E\left[\left|e_{r}(k)\right|^{2}\right] .
$$

Thus, minimizing $E\left[|e(k)|^{2}\right]$ is equivalent to minimize $E\left[\left|e_{d}(k)\right|^{2}\right]$. Thus, we can use the PES, $e$, as a corrupted error signal to do the adaptation, where $e_{r}$ acts as the measurement noise for the adaptation process.

The regressor defined in Eq. (10) will be estimated by filtering $z$ with the MA model, $\hat{G}_{M A}$.

$$
\hat{x}(k)=\hat{G}_{M A}\left(q^{-1}\right) z(k) .
$$

The recursive least square (RLS) PAA for tuning the tap weights of $H$ is (Åström and Wittenmark, 1995)

$$
\begin{aligned}
\hat{\theta}(k) & =\hat{\theta}(k-1)+P(k) \phi(k) e(k-1), \\
P(k) & =P(k-1) \\
& -\frac{P(k-1) \phi(k-1) \phi^{T}(k-1) P(k-1)}{1+\phi^{T}(k-1) P(k-1) \phi(k-1)}(19)
\end{aligned}
$$


Simulations were conducted using a MEMS MA dual-stage model. The dual-stage feedback controller was designed using the sensitivity decoupling method described in ( $\mathrm{Li}$ and Horowitz, $2001 b$ ). The sampling frequency was assumed to be $40 \mathrm{kHz}$. The designed dual stage feedback controller has an open loop cross-over frequency of $4.6 \mathrm{kHz}$. Two suspension resonance modes respectively at 6.7 and $8.6 \mathrm{kHz}$ are included in the simulation. $G_{w d}$ and $G_{w z}$ in Fig. 4 have the same poles, but slightly different zeros. Runout, disturbances, and measurement noises are injected into the plant at corresponding locations in the simulations.

Fig. 5 shows the FFT of the PES when feedforward vibration cancellation is not applied. Fig. 6 shows the FFT of the PES when adaptive feedforward control is applied, and after the control parameters have converged. The FIR filter is chosen to be 6 -tap. We can see that the adaptive feedforward controller for the MA can successfully suppress the suspension vibration induced TMR, as evidenced by the significant reduction in the magnitude of the FFT peaks in the $6-12 \mathrm{kHz}$ frequency range.

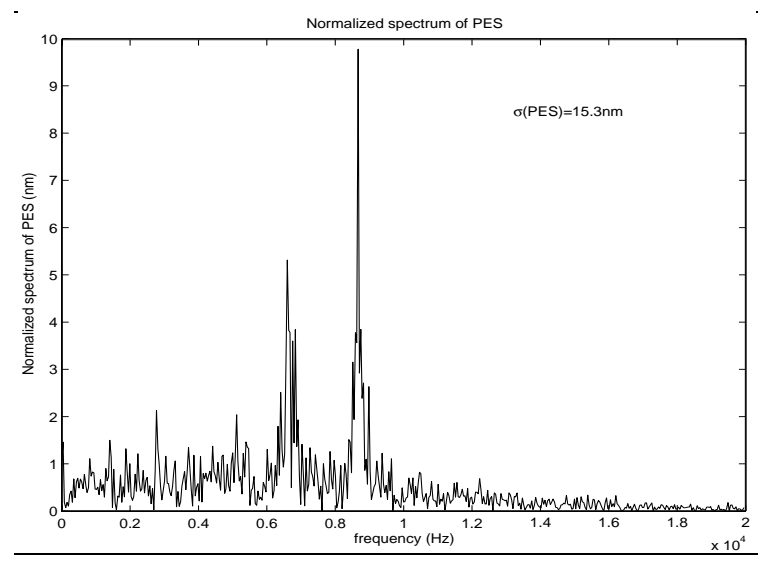

Fig. 5. FFT of PES without feedforward control

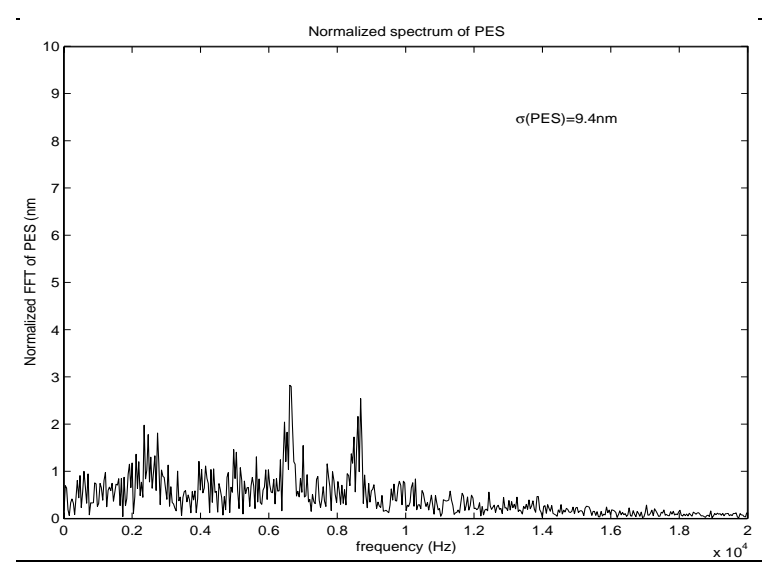

Fig. 6. FFT of PES with adaptive feedforward control
A prototype MEMS microactuator for computer dual-stage servo system was designed and fabricated. The use of structural electrical-isolation in the MA design considerably reduces the driving voltage. High gauge factor vibration strain sensors was fabricated by depositing polysilicon on a metal wafer. Simulation showed adaptive feedforward control was effective in compensating the suspension vibration induced TMR for actuated slider dual-stage servo system with a rotational microactuator. Future work includes finishing the fabrication of the microacutator and the vibration sensor, and experimental testing of the vibration control scheme.

\section{REFERENCES}

Åström, K. J. and B. Wittenmark (1995). Adaptive Control. 2nd ed.. Addison-Wesley.

Fan, L.-S., T. Hirano, J. Hong, P. R. Webb, W.H. Juan, W. Y. Lee, S. Chan, T. Semba, W. Imaino, T.S. Pan, S. Pattanaik, F.C. Lee, I. McFadyen, S. Arya, and R. Wood (1999). Electrostatic mciroactuator and design considerations for hdd application. IEEE Transactions on Magnetics 35, 1000-1005.

Horsley, D., N. Wongkomet, R. Horowitz and A. Pisano (1999). Precision positioning using a microfabricated electrostatic actuator. IEEE Transactions on Magnetics 35, 993999.

Huang, Y., M. Banther, P.D. Mathur and W.C. Messner (1999). Design and analysis of a high bandwidth disk drive servo system using an instrumented suspension. IEEE/ASME transaction of Mechatronics 4, 196-206.

Li, Y. and R. Horowitz (2001a). Active suspension vibration control with dual stage actuators in hard disk drives. Proceedings of American Automatic Control Conference.

Li, Y. and R. Horowitz (2001b). Mechatronics of electrostatic microactuator for computer disk drive dual-stage servo systems. IEEE/ASME transaction of Mechatronics 6, 111-121.

Li, Y. and R. Horowitz (2002). Active vibration control of a pzt actuated suspension in hard disk drives. Proceedings of American Automatic Control Conference.

Muller, L. (2000). Ph.D. Thesis: Gimballed Electrostatic Microactuators with Embedded Interconnects. University of California. Berkeley, CA, USA. 